李飞飞,高珂晓,朱金方,柳晓燕,赵彩云.综合物理防控技术对盐城大丰港互花米草的控制效果.生态学报,2021,41(24):9637-9644.

Li F F, Gao K X, Zhu J F, Liu X Y, Zhao C Y.Effect of comprehensive physical control technologies on Spartina alterniflora in Dafeng Port, Yancheng. Acta Ecologica Sinica, 2021,41(24):9637-9644.

\title{
综合物理防控技术对盐城大丰港互花米草的控制效果
}

\author{
李飞飞,高珂晓,朱金方,柳晓燕,赵彩云* \\ 中国环境科学研究院,环境基准与风险评估国家重点实验室, 北京 100012
}

\begin{abstract}
摘要:互花米草是我国沿海滩涂典型外来人侵物种之一,该物种在江苏盐城大丰港形成了大面积人侵种群。在大丰港设置了刈 割+遮荫和单纯刚割样方共 9 个,对比分析了不同防控技术以及不同遮荫持续时间对互花米草地上部分和地下根茎的影响,评 估遮荫去除后互花米草生长状况、土壤氮磷含量、底栖动物生物量和密度的变化,以探索适合的控制技术和有效控制时间。结 果表明:两种控制技术都极显著降低了互花米草地上部分株高、基径、密度和地上鲜重 $(P<0.01)$,对已刈割的互花米草遮荫 3 个月、6个月、9个月和 12 个月后依次去除遮荫网, 互花米草地上部分均全部消失, 直至第二个生长季均未重新长出幼苗; 两种 控制技术显著降低了互花米草地下根茎存活率 $(P<0.05)$, 但控制技术之间和遮荫时间长短对根茎存活率的影响无显著差异; 去除遮荫网后, 底栖动物生物量显著增加 $(P<0.05)$, 但土壤氮磷含量无显著变化。研究发现在江苏盐城大丰港实施刈割 + 遮荫 3 个月后去除遮荫网, 是治理互花米草、恢复本土动植物最高效的防控方法。
\end{abstract}

关键词:互花米草;防控技术;遮荫;根茎成活率; 大型底栖动物

\section{Effect of comprehensive physical control technologies on Spartina alterniflora in Dafeng Port, Yancheng}

LI Feifei, GAO Kexiao, ZHU Jinfang, LIU Xiaoyan, ZHAO Caiyun *

State Key Laboratory of Environmental Criteria and Risk Assessment, Chinese Research Academy of Environmental Sciences, Beijing 100012 , China

\begin{abstract}
Spartina alterniflora is one of the typical invasive alien species along the coast of China. This species has formed a large-scale invasive population in Dafeng Port, Yancheng, Jiangsu. This study set up a total of 9 mowing +shading and only mowing plots in Dafeng Port and analyzed the differences of influences on the upper part and underground rhizomes of S. alterniflora among different control technologies and different lengths of shading time. We further monitored the control effect of $S$. alterniflora and the impact on soil nitrogen and phosphorus content and macrobenthos after removing the shading net. To explore the shortest shading time required to effectively control Spartina alterniflora. The results showed that the two control technologies extremely significantly reduced the plant height, base diameter, density, and fresh weight of the aboveground plant of $S$. alterniflora $(P<0.01)$. After mowing+shading was implemented for 3, 6, 9, and 12 months, the shading net was removed in turn, the aboveground part of $S$. alterniflora disappeared completely, and did not re-grow at the vigorous growth period of the second year. Both of the two control techniques significantly reduced the survival rates of the rhizomes of $S$. alterniflora $(P<0.05)$. And there was no significant difference among the effects of different control technologies and different lengths of shading time on the survival rate of rhizomes. After removing the shading net, the biomass of macrobenthos increased significantly $(P<0.05)$, but the soil nitrogen and phosphorus content did not change significantly. This study found that removing the shading net after 3 months of mowing+shading control technology in Dafeng
\end{abstract}

基金项目: 国家重点研发计划项目(2017YFC0506200); 生态环境部生物多样性调查评估项目(2019HJ2096001006)

收稿日期:2021-03-02; 采用日期:2021-07-20

*通讯作者 Corresponding author.E-mail: zhaocycraes@126.com, zhaocy@ craes.org.cn 
Port, Yancheng, Jiangsu Province, is the most efficient prevention and control method for treating Spartina alterniflora and the restoration of native species.

Key Words : Spartina alterniflora; control technology; shading; the survival rate of rhizomes; macrobenthos

互花米草 (Spartina alterniflora) 隶属于禾本科米草属,原产于北美洲与南美洲的大西洋沿岸, 现已人侵到 世界各地沿海地区 ${ }^{[1-2]}$ 。1979 年, 我国从美国北卡罗来纳州、佐治亚州、佛罗里达三地引入该物种 ${ }^{[3-4]}$, 并于 1981 年在罗源湾试种成功后推广至我国沿海各省 ${ }^{[5]}$ 。目前, 互花米草分布区域北至河北唐山,南达广西北 海, 遍布我国沿海海岸, 总面积达 $54579.7 \mathrm{hm}^{2[6]}$ 。该外来物种的迅速扩散蔓延, 对我国海岸带生态系统和生 物多样性保护造成一定威胁 ${ }^{[7-8]}$ 。2003 年,生态环境部将其列人中国第一批外来入侵物种名单中 ${ }^{[1]}$ 。

1982 年起, 江苏省在启东、射阳、滨海、灌云、连云港、赣榆等地成功种植互花米草, 随后互花米草种群在 江苏沿海海岸迅速扩张, 形成大片的互花米草盐沼 ${ }^{[9]}$ 。至今, 互花米草在该省分布面积达到 $18363 \mathrm{hm}^{2[6]}$ 。 张华兵等 ${ }^{[10]}$ 通过对盐城滨海湿地生境变化分析, 预计至 2030 年, 互花米草沼泽在不受控制的状态下面积百 分比将扩张至盐城珍禽保护区核心区的 $64.06 \%$ 。互花米草不仅大面积侵占了芦苇、碱蓬、白茅等本地植物物 种的生态位 ${ }^{[10-11]}$, 并且严重影响了乌类栖息地、鸟类群落格局 ${ }^{[12]}$ 、麘鹿栖息地、麇鹿取食结构 ${ }^{[13]}$ 。因此,有效 控制互花米草的扩张, 并进一步开展防控治理已成为保护江苏省滨海湿地生态系统的关键问题之一。

目前, 治理外来人侵植物的方法主要分为化学方法和非化学方法。以喷酒除草剂为主的化学控制对加拿 大一枝黄花等外来入侵植物有很好的杀伤效果 ${ }^{[14]}$, 但对本地物种 ${ }^{[15-16]}$ 和生态环境都具有一定的负面影响, 尤 其是在湿地生态系统中, 研究者更推荐使用非化学方法 ${ }^{[17]}$ 。Weidlich 等 ${ }^{[18]}$ 分析了近 20 年发表的 372 篇文 献, 发现非化学方法中使用最多的是刈割和火烧。刚割是欧洲国家治理路边豚草的首选方法 ${ }^{[19]}$, 也同样是人 侵北美的燕麦草 (Arrhenatherum elatius $)^{[20]}$ 和人侵欧洲的多叶羽扇豆 (Lupinus polyphyllus) ${ }^{[21]}$ 的主要治理方 法。而火烧多用于控制沙漠、温带针叶林和落叶林以及温带草原中的外来人侵物种 ${ }^{[18]}$ 。但单纯的刈割和火 烧都只能去除入侵植物的地上部分, 不能阻止地下部分的再次萌发 ${ }^{[22-23]}$ 。

常用的互花米草物理防治方法包括刈割、遮荫、水淹等 ${ }^{[24-26]}$, 但互花米草具有强大的无性繁殖能力, 在地 上部分清除或死亡后, 地下根茎仍然具有很强的萌发能力 ${ }^{[27-28]}$ 。Zhao 等发现单纯刈割的治理方式无法抑制 广西北海互花米草再次萌发, 但“刘割+遮荫” 处理后的第二个生长季末, 互花米草无性繁殖个体或幼苗就已 完全消失, 能有效治理互花米草 ${ }^{[29]}$ 。虽然前期研究还探索了“刈割+遮荫” 控制方法中遮荫强度和遮荫时长, 但这些研究仅监测了遮荫实施期间互花米草的存活状况, 对去除遮荫网后互花米草控制效果还未见报道。

因此,为探索有效控制互花米草的技术方法,并能进一步开展实际应用示范,本研究在江苏盐城大丰港实 施了“刈割” 和“刈割+遮荫”物理防控技术, 在不同时期去除遮荫网后定期监测互花米草地上部分和地下根茎 的存活状况，分析了不同防控技术和不同遮荫时间长度对样地土壤氮磷含量和底栖动物的影响。以探明不同 治理技术对互花米草的实施效果, 并探索实现有效控制互花米草所需的最短遮荫时间, 为江苏盐城大丰港互 花米草的控制篮选最优治理技术方案。

\section{1 研究区域与方法}

\section{1 研究区域概况}

本研究在江苏省盐城大丰港大坝附近 $\left(33.0554^{\circ} \mathrm{N}, 120.8594^{\circ} \mathrm{E}\right)$ 开展, 实验所在区域,互花米草成大面积 分布,互花米草种群边缘,生长着少量盐地碱蓬, 该区域常有鹿鹿和人类活动干扰。

\section{2 实验方法}

2018 年 5 月, 在互花米草斑块的中间位置设置了 1 个 $625 \mathrm{~m}^{2}$ 的样区, 将样区内互花米草进行刈割处理, 刈割时保证留茬不超过 $3 \mathrm{~cm}$, 在样区内随机布设 9 个 $6 \mathrm{~m} \times 6 \mathrm{~m}$ 处理样方 (图 1), 样区周围 $10 \mathrm{~m}$ 范围内为不进 
行任何处理的对照样地。基于前期研究结果,3 层遮荫网平均透光率最低,仅有 $0.31 \%{ }^{[30]}$ 。因此,本研究每个 处理样方采用 3 层 $6.2 \mathrm{~m} \times 6.2 \mathrm{~m}$ 大小的遮荫网覆盖,遮荫网拉紧后使用木楔子固定四角及中线(图 2)。将 6 个 $6 \mathrm{~m} \times 6 \mathrm{~m}$ 样方从中间分割成 12 个 $3 \mathrm{~m} \times 6 \mathrm{~m}$ 的处理样方, 遮荫持续时长设置为 3 个月、 6 个月、 9 个月、 12 个 月, 每种遮荫持续时间设置 3 个重复, 去除遮荫网时间分别为 2018 年 8 月 (遮荫 3 个月)、2018 年 11 月 (遮荫 6 个月)、2019年 2 月 (遮荫 9 个月)、2019 年 5 月(遮荫 12 个月)。

\section{3 指标测定}

\subsection{1 互花米草地下、地上部分监测}

在去除遮荫网的同时调查相应样方地下根茎存活 率, 以反映不同控制技术及控制时间长度对地下根茎的 影响。在第二个生长季末 ( 2019 年 11 月) 再次调查所 有处理样方及无处理对照样地地下根茎存活率, 以监测 去除遮荫网后地下根茎活力是否恢复。采用土钻法挖 取刈割+遮荫样方、单纯刚割样方及无处理对照样地中 $25 \mathrm{~cm}$ 深的土壤 3 份 (钻头直径 $3.8 \mathrm{~cm}$, 每 3 钻为一份 带土根茎样品), 海水冲掉泥沙后, 根据根表面和根断 面颜色分辨死根茎和活根茎 ${ }^{[24,31]}$, 烘干后称量干重, 计 算地下根茎存活率, 根茎存活率计算公式为:

$$
\text { 地下根茎存活率 }=\frac{\text { 活根茎干重 }}{\text { 活根茎干重 }+ \text { 死根茎干重 }}
$$

地上部分调查时间为互花米草第二个生长季的生 长旺盛期 ( 2019 年 7 月), 调查时在刈割 + 遮荫样方、单 纯刈割样方及无处理对照样地互花米草种群中随机设 置 3 个 $0.5 \mathrm{~m} \times 0.5 \mathrm{~m}$ 的小样方进行调查。统计小样方 内互花米草株数, 并从每个小样方中随机选取 3 株个 体, 测量每株株高和基径, 称量 10 株地上部分鲜重。

\subsection{2 土壤氮磷含量监测}

2019 年 5 月,在每个刚割+遮荫和单纯刘割处理样 方内用土钻法采集样地土壤样品 3 份 (钻头直径 3.8 $\mathrm{cm}$ 、长 $25 \mathrm{~cm}$, 每 3 钻为一份带土根茎样品), 另在无处 理互花米草种群中采集 3 份土壤样品作为对照,测定土 壤全氮和总磷含量, 全氮采用凯氏法测定, 总磷采用碱 熔-钼锑抗分光光度法测定。

\subsection{3 底栖动物监测}

2020 年 7 月,在刈割+遮荫处理样方、单纯刚割处

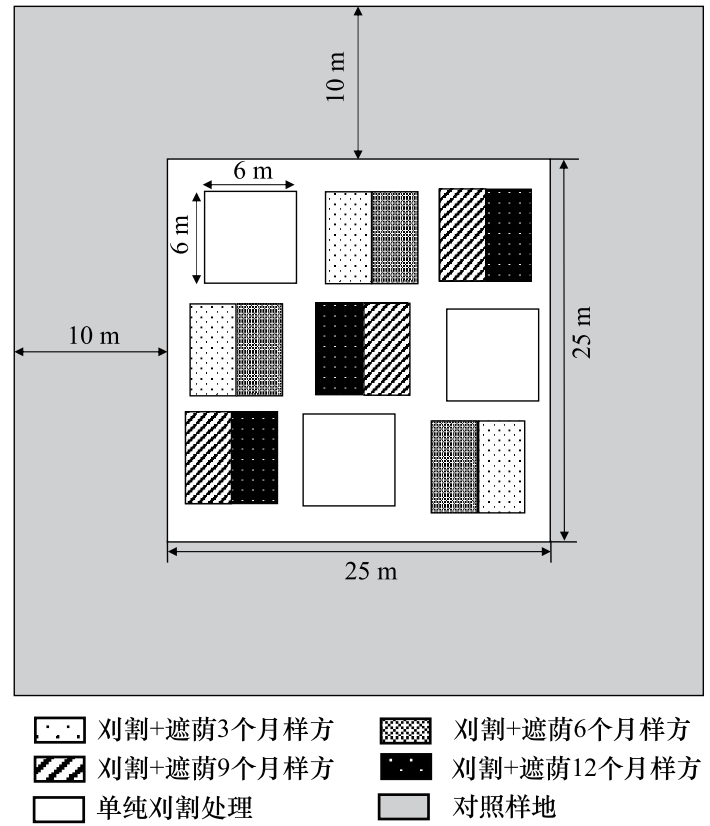

图 1 控制技术样方设置

Fig.1 Quadrats of different control technology

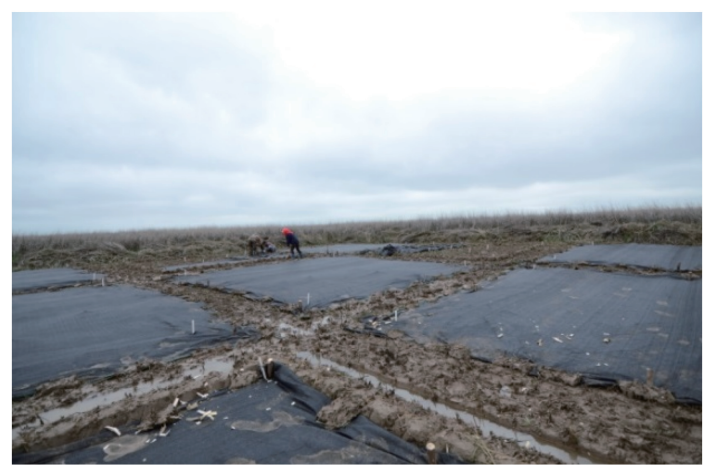

图 2 刈割+遮荫控制技术实施

Fig.2 Mowing+shading control technology 理样方以及无处理互花米草种群中心位置随机采集 3 份样品, 采样方法为: 使用钻头直径 $7 \mathrm{~cm}$ 的土钻采样, 采样深度 $25 \mathrm{~cm}$, 每个采样点采集 3 钻合为一份样品, 采样面积 $115 \mathrm{~cm}^{2}$ 。将所采泥样用 $0.5 \mathrm{~mm}$ 孔径的分样篮进行淘洗, 获取大型底栖动物样品, 并用 $75 \%$ 酒精装 瓶保存,实验室内分拣、种类鉴定、计数并称量。

\section{4 统计分析}

为检验不同处理间互花米草各指标的差异,经过方差齐性 (Levene's test) 检验后,采用单因素方差分析 (one-way ANOVA) 对样本数据进行分析。方差分析 $F$ 检验显著 $(P<0.05)$ 时,采用 Tukey 进行多重比较分 
析。对不同遮荫时长根茎存活率的对比分析采用双因素重复测量方差分析 ( Two-way repeated-measures ANOVA) ,数据分析使用 SPSS 26 进行。

\section{2 结果与分析}

2.1 不同控制技术及控制时间长度对地上部分特征的影响

刈割+遮荫 3 个月起, 互花米草地上部分就已全部 消失 ( 图 3), 在第二个生长季生长旺盛期 (2019 年 7 月) 调查发现, 刈割+遮荫样方内的互花米草均未重新 长出,而刈割样方内互花米草重新萌发生长。单因素方 差分析结果显示刈割+遮荫、单纯刈割以及对照的互花 米草地上高度和基径都存在极显著差异 $(P<0.01)$,但 刈割+遮荫和单纯刚割处理对互花米草密度和地上部 分鲜重的影响无明显差异 $(P>0.05)$ (图 4)。

2.2 不同控制技术及控制时间长度对地下部分特征的 影响

控制处理 3 个月后, 刈割+遮荫和单纯刈割处理地

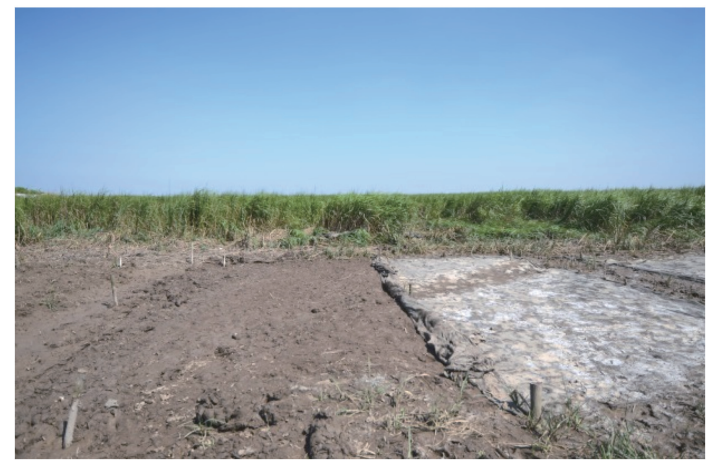

图 3 刈割 + 遮荫控制技术实施 3 个月后互花米草地上部分

Fig.3 The above-ground parts of $S$. alterniflora after mowing + shading three months ago 下根茎存活率分别为 $25.65 \%$ 和 $41.84 \%$, 低于无处理对 照 $73.70 \%$; 控制处理 6 个月后, 两种控制处理地下根茎存活率有所上升, 分别为 $42.25 \%$ 和 $59.87 \%$, 无处理对 照的根茎存活率反而有所下降,为 $64.33 \%$; 控制处理 9 个月后, 单纯刈割处理的根茎存活率为 $64.42 \%$, 非常 接近无处理对照的根茎存活率 $68.24 \%$, 刈割 + 遮荫处理的根茎存活率也有所上升, 但仍然是最低的, 为 $57.54 \%$; 控制处理一年后, 单纯刈割处理的根茎存活率下降较多,与刚割+遮荫处理几乎相同, 分别为 $45.98 \%$ 和 $44.37 \%$, 无处理对照为 58.99\% (图 5)。重复测量方差分析结果表明, 控制时间长度对地下根茎存活率没 有显著影响 $(P>0.05)$, 但控制技术对地下根茎存活率具有显著影响 $(P<0.05)$, 控制技术和控制时间长度的交 叉影响不显著 $(P>0.05)$ (表 1$)$ 。

表 1 不同控制技术和不同时间及其交互作用对互花米草根茎存活率影响的双因素方差分析表

Table 1 Two-way ANOVA on the effects of different control technologies, different time and their interaction on Rhizome survival rate of S. alterniflora

\begin{tabular}{lcc||lc}
\hline 因子 Factors & $F$ & $P$ & 因子 Factors & $P$ \\
\hline 控制技术 Control technologies & 20.346 & 0.008 & 控制技术×时间 & 2.126 \\
时间 Time & 3.708 & 0.081 & Control technologies×Time & 0.121 \\
\hline
\end{tabular}

在第二个生长季末, 无处理对照样方地下根茎平均存活率为 $59.74 \%$, 单纯刚割样方平均存活率为 $30.19 \%$,刏割+遮荫样方存活率在 $31.18 \%-38.62 \%$ 之间。与无处理对照相比, 刈割+遮荫和单纯刈割技术对 互花米草地下根茎均具有显著影响 $(P<0.05)$, 但两种控制方式以及不同遮荫持续时间对互花米草地下根茎 的影响无显著差异 $(P>0.05)$ ( 图 6)。

\section{3 不同控制技术对土壤氮磷的影响}

通过对比刈割+遮荫不同时间长度、单纯刈割以及无处理对照的土壤全氮和总磷含量发现,经过一年的 治理,未见对土壤氮磷的影响,所有处理和对照全氮和总磷均无明显差异 (图 7)。

2.4 不同控制技术对底栖动物的影响

2020 年 7 月在刈割+遮荫、单纯刚割控制技术以及无处理对照的样方中共采集到三种底栖动物, 分别是 腹足纲 (Gastropoda) 汇螺科 (Potamididae) 的珠带拟蟹守螺 (Cerithideopsilla cingulata) 和拟沼螺科 (Assimineidae) 的绯拟沼螺 (Assiminea latericea), 以及双壳纲 (Bivalvia) 绿螂科 (Glaucomyidae) 的中国绿螈 

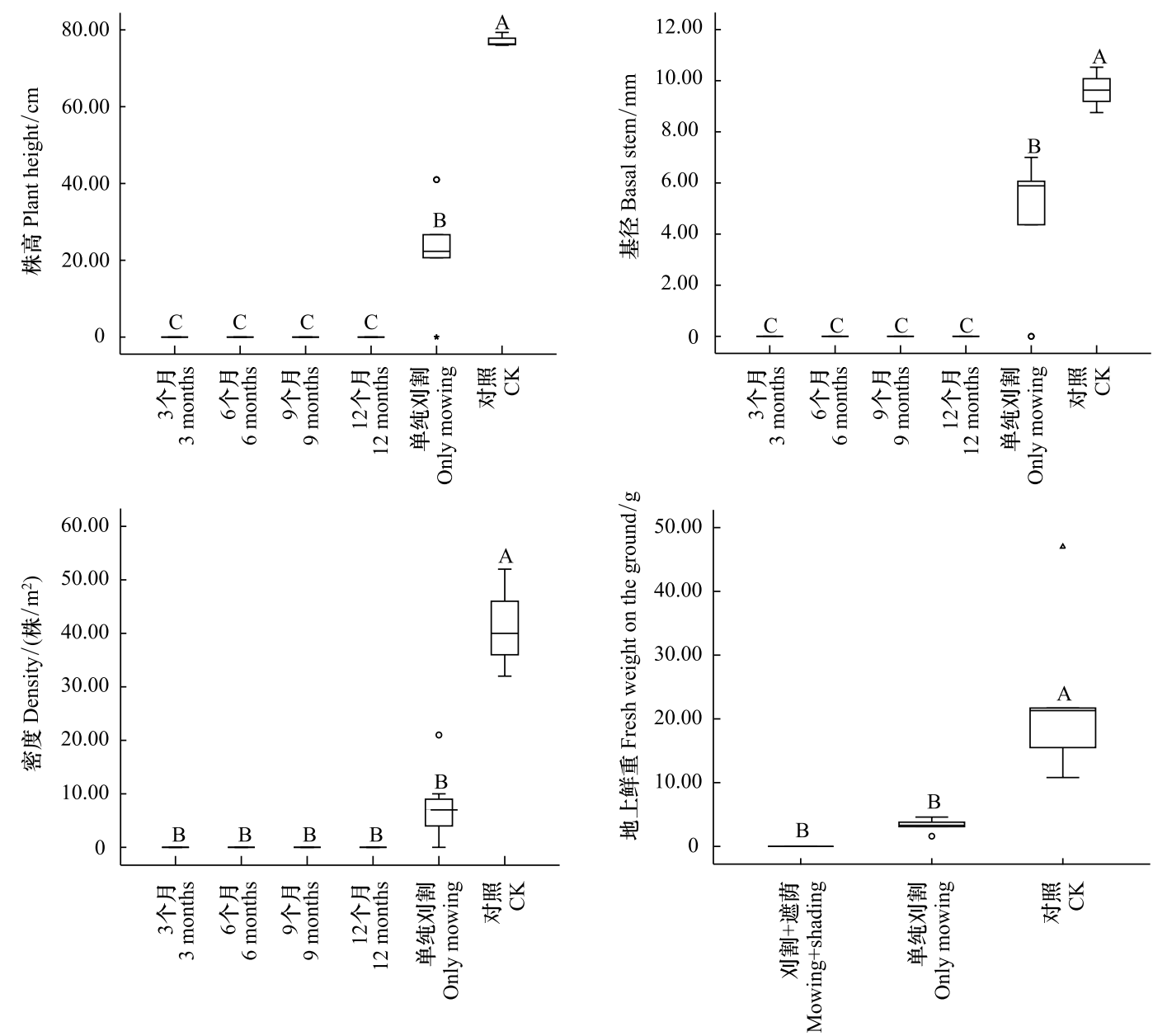

图 4 不同遮荫时间长度对互花米草地上部分的影响

Fig.4 The effect of different lengths of shading time after mowing on the growth characteristics of above-ground parts of $S$. alterniflora 不同大写字母代表极显著差异 $(P<0.01)$

(Glaucomya chinensis)。不同控制技术和无处理对照样 方中均有这三种底栖动物, 基于方差分析结果表明,两 种控制技术和无处理对照中底栖动物密度无显著差异 (图 8); 刏割+遮荫控制技术实施后底栖动物生物量显 著高于无处理对照 $(P<0.05)$, 单纯刈割控制技术实施 后底栖动物生物量与刈割 +遮荫控制技术和无处理对 照均无显著差异(图 8)。

\section{3 讨论}

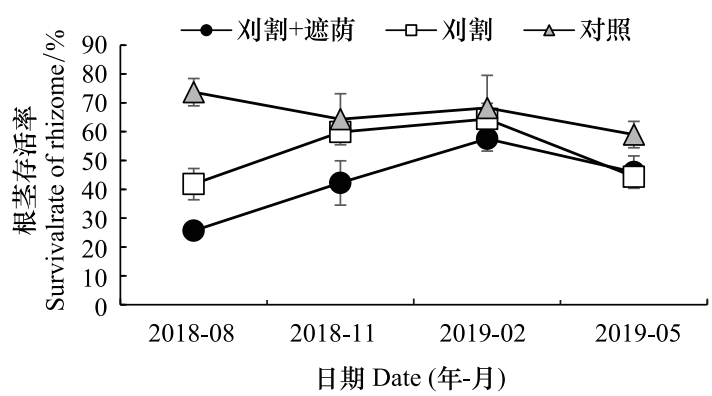

图 5 不同控制技术在不同时间段地下根茎的存活率

在互花米草的防控治理中,综合防控技术比单一技 术能够取得更好的控制效果 ${ }^{[32]}$ 。本研究对比了刘割+ Fig.5 Rhizome survival rate of $S$. alterniflora in different control technologies at different time 遮荫和单纯刚割两种控制技术对江苏盐城大丰港互花 米草种群的防治效果,监测了不同控制技术和控制时间长度对互花米草地上和地下部分、土壤理化性质以及 底栖动物的影响。

研究表明,在该区域实施刈割+遮荫控制技术后对互花米草地上部分具有迅速有效的控制效果,遮荫 3 
个月互花米草地上部分已全部清除。这与前期研究结 果一致,赵相健等对广西北海互花米草实施刈割+遮荫 防控技术, 2 个月后地上部分已全部消失 ${ }^{[30]}$ 。但张丽 平等在福建省霞浦县实施则割+遮荫处理发现, 如果不 贴地覆盖, 给互花米草较大的生存空间, 即便遮荫强度 很高, 在 3 个月后也无法彻底清除互花米草地上部 分 ${ }^{[33]}$ 。Zhao 等发现, 在 5 月和 7 月实施刘割+遮荫防控 技术比在 8 月和 10 月实施效果好 ${ }^{[29]}$ 。因此, 刈割时间 和遮荫方式的不同, 控制效果也会有所差异。本研究所 设置的刈割时间为 5 月,采用 3 层遮荫网贴地覆盖遮 荫,互花米草生存空间小,有效降低了互花米草可溶性 糖和可溶性蛋白含量, 从而降低互花米草光合作用效 率, 抑制地上部分生长 ${ }^{[33-34]}$ 。

防控技术实施后, 人侵物种是否再次萌发或二次人 侵是评估该防控技术成效的关键，前期研究均没有进一 步监测去除遮荫网后互花米草生长状况。本研究设置

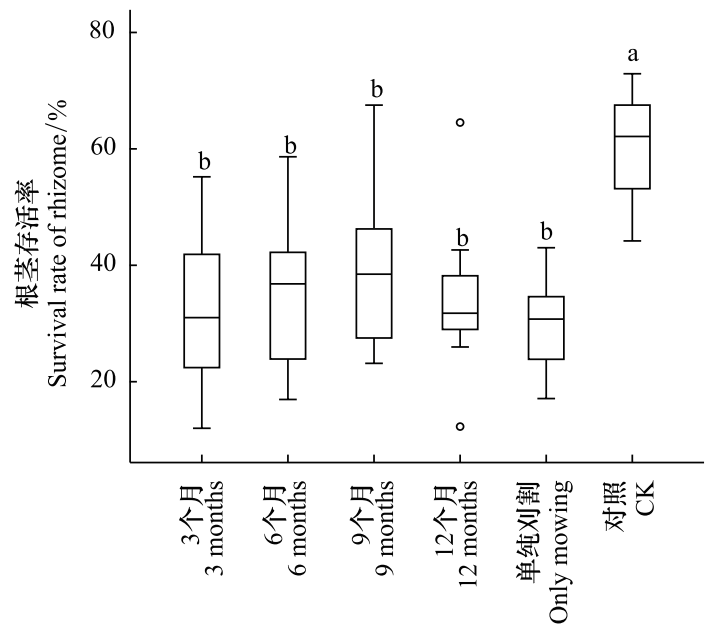

图 6 不同遮荫时间长度对互花米草根茎存活率的影响

Fig.6 The effect of different lengths of shading time after mowing on rhizome survival rate of $S$. alterniflora 不同小写字母代表显著差异 $(P<0.05)$ 了不同遮荫持续时间,并在完成后去除遮荫网, 结果发 现, 所有则割+遮荫样方内的互花米草地上部分一直没有恢复, 直至第二个生长季也未长出, 而单纯刈割控制 效果不佳,在第二个生长季内地上部分重新萌发生长。
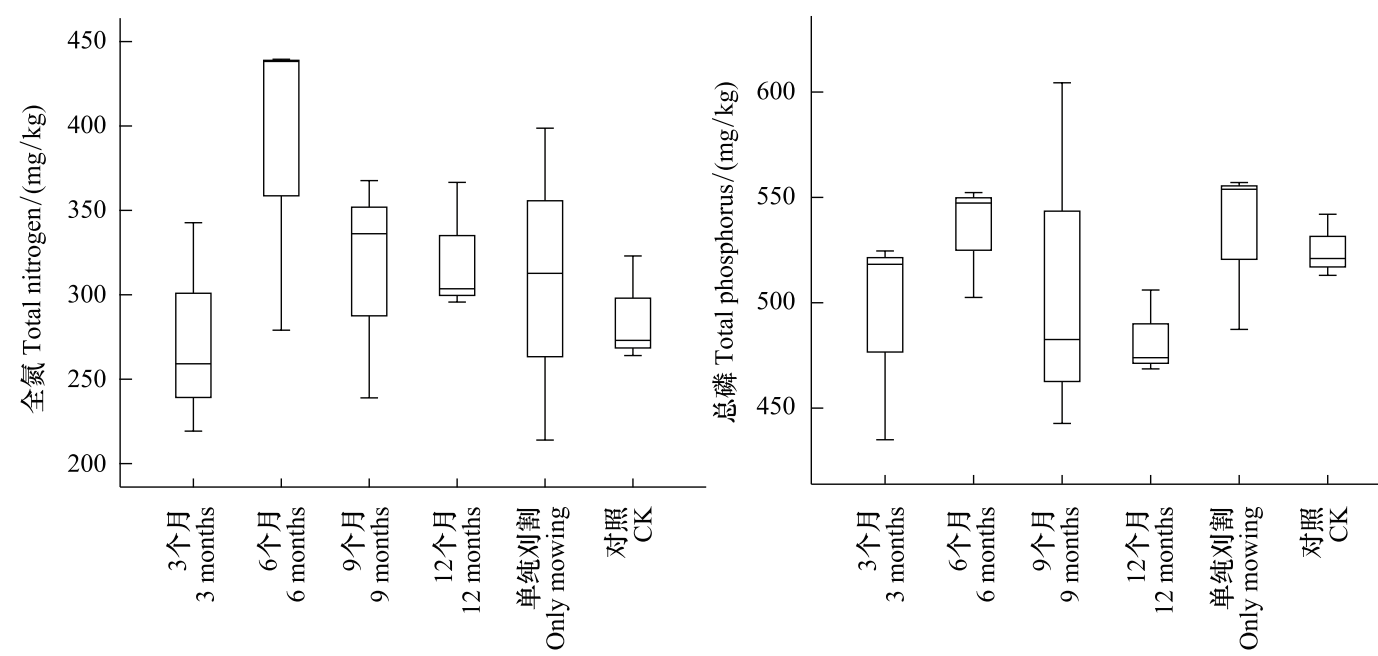

图 7 不同遮荫时间长度对土壤理化性质的影响

Fig.7 The effect of different lengths of shading time after mowing on soil total nitrogen and total phosphorus

此外,刚割+遮荫和单纯刈割对互花米草地下根茎的影响要显著高于无处理对照,但不同遮荫持续时间 和是否遮荫对互花米草地下根茎的影响差异不大。刚割+遮荫和单纯刚割两种控制技术在不同时间段对地 下根茎的影响表现为地下根茎存活率先急剧降低,随后逐渐升高又下降的过程。推测这是由于互花米草生长 期过后营养物质逐渐向下转移到根茎,到了萌发期根茎活力升高, 至第二个生长季末, 所有样方已去除遮荫网 6 至 15 个月,互花米草地上部分仍没有重新萌发, 失去了原有地上部分光合作用所提供的物质和能量, 同时 地下部分的物质和能量储备较小, 地下根茎开始逐渐死亡。本研究中地下根茎存活率要高于赵相健等 ${ }^{[30]}$ 对 广西北海互花米草种群实施刈割+遮荫后的根茎存活率, 推测这是由于分布于我国中部地区的互花米草较广 

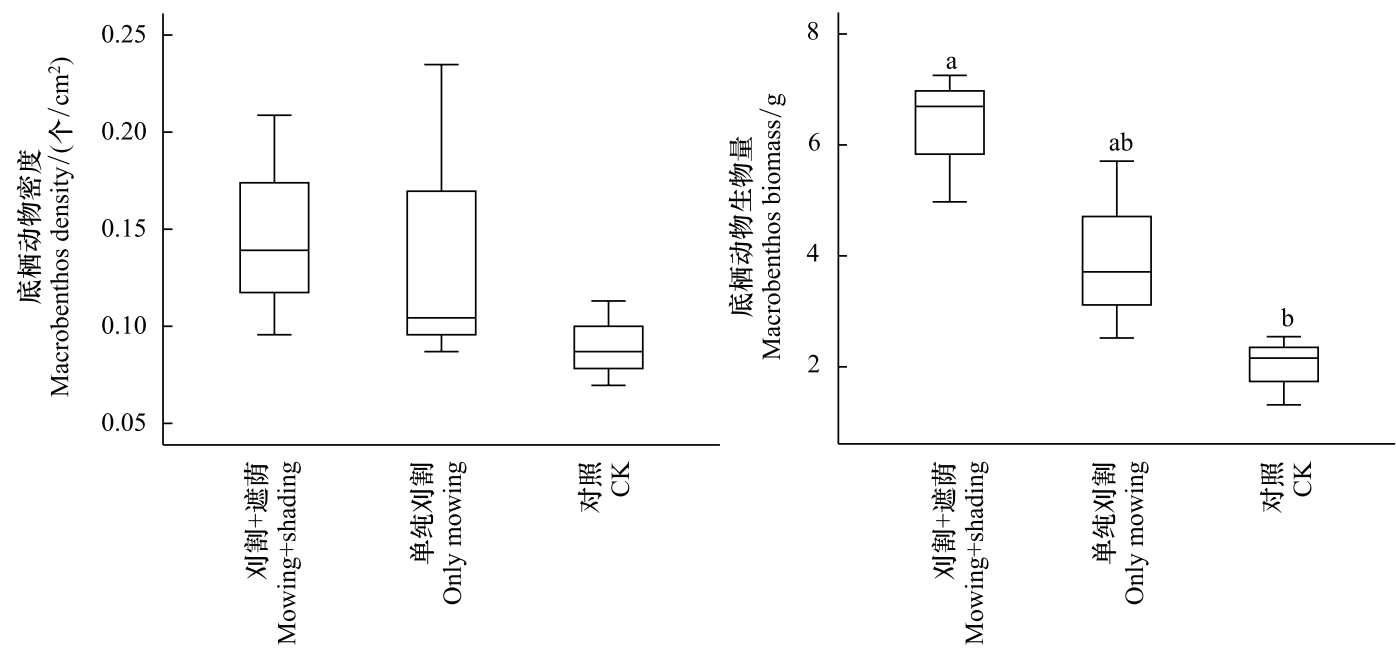

图 8 不同控制技术对底栖动物总栖息密度和生物量的影响

Fig.8 Density and biomass of macrobenthos in different control technologies

不同小写字母代表显著差异 $(P<0.05)$

西北海的更高大,且基径更粗 ${ }^{[1]}$,地下根茎生长活力可能更高。

本研究发现,经过刈割+遮荫控制技术的实施,在去除遮荫网后, 底栖动物生物量显著增加。单纯刘割技 术虽也增加了底栖动物生物量,但效果并不显著。对比前期研究发现, 在不同区域内,互花米草人侵对底栖动 物的影响有所不同。赵彩云等 ${ }^{[35]}$ 发现互花米草的人侵增加了大型底栖动物的物种数、生物量和总栖息密度。 而仇乐等 ${ }^{[36]}$ 发现随着互花米草人侵江苏射阳河口的时间增加, 底栖动物的数量和多样性下降。但两种控制 技术短期内对土壤氮磷含量影响并不明显。

互花米草遍布我国沿海海岸,但由于我国海岸线较长,气候环境因子差异很大,针对地理位置不同的互花 米草种群进行治理，应当综合考虑治理成本、治理时间及治理效果，从而篮选最佳控制治理技术,采取因地制 宜的控制管理办法。例如, 在广东、广西以及福建沿海滩涂红树林分布区, 可采用种植红树植物生物替代互花 米草的方法治理 ${ }^{[37-39]}$ 。但由于江苏盐城滨海湿地不在红树林自然分布区,这种方法并不适用。本研究在江 苏盐城大丰港实施控制技术后发现,刈割+遮荫技术比单纯刈割对当地互花米草治理更为彻底, 刈割+遮荫防 控技术实施 3 个月后便可以去除遮荫网，从达到高效治理互花米草、自然恢复本土动植物的目的。

\section{参考文献 (References) :}

［1］赵彩云, 李俊生, 赵相健. 中国沿海互花米草人侵与防控管理. 北京: 科学出版社, 2015.

[ 2 ] Li F F, Gong L, Li J S, Liu X Y, Zhao C Y. Low genetic differentiation yet high phenotypic variation in the invasive populations of Spartina alterniflora in Guangxi, China. PLoS One, 2019, 14(9): e0222646.

[ 3 ] Bernik B M, Li H S, Blum M J. Genetic variation of Spartina alterniflora intentionally introduced to China. Biological Invasions, 2016, 18(5) : 1485-1498.

[ 4 ] Deng Z F, An S Q, Zhou C F, Wang Z S, Zhi Y B, Wang Y J, Shi S H, Chen L, Zhao C J. Genetic structure and habitat selection of the tall form Spartina alterniflora Loisel. in China. Hydrobiologia, 2007, 583(1) : 195-204.

[ 5 ] 徐国万, 卓荣宗, 曹豪, 李相敢. 互花米草生物量年动态及其与滩涂生境的关系. 植物生态学报, 1989, 13(3) : 230- 235.

[ 6 ] 刘明月. 中国滨海湿地互花米草人侵遥感监测及变化分析 $[D]$. 长春: 中国科学院大学 (中国科学院东北地理与农业生态研究所), 2018.

[ 7 ] 潘卫华, 陈家金, 王岩. 近 20 年福建红树林和互花米草群落时空变化及景观特征. 生态与农村环境学报, 2020, 36(11): 1428-1436.

[8] 王卿, 安树青, 马志军, 赵斌, 陈家宽, 李博. 人侵植物互花米草一一生物学、生态学及管理. 植物分类学报, 2006, 44(5): 559-588.

[ 9 ] 沈永明, 刘咏梅, 陈全站. 江苏沿海互花米草 (Spartina alterniflora Loisel) 盐沼扩展过程的遥感分析. 植物资源与环境学报, 2002, 11(2)： 33-38.

[10］张华兵, 高卓, 王娟, 刘玉卿, 韩爽, 张亚楠, 李玉风. 基于“格局-过程-质量” 的盐城滨海湿地生境变化分析. 生态学报, 2020, 40(14)： 
4749-4759.

[11] 王娟, 张华兵, 刘玉卿, 韩爽, 徐雅, 张亚楠. 盐城滨海湿地植物群落种间格局与竞争的关系研究. 生态学报, 2020, 40(24): 8966-8973.

[12] 张燕, 孙勇, 鲁长虎, 张亚兰, 吕士成. 盐城国家级珍禽自然保护区互花米草人侵后三种生境中越冬鸟类群落格局. 湿地科学, 2017, 15 (3): 433-441.

［13］滑荣, 崔多英, 刘佳, 李淑红, 赵永强, 张亚楠. 江苏盐城湿地麇鹿冬季食性研究. 动物学杂志, 2020, 55(1): 1-8.

[14] 查国贤, 张国彪, 徐建方, 郝建华. 加拿大一枝黄花的防控措施. 杂草科学, 2011, 29(4): 46-49.

[15] Holl K D, Howard E A, Brown T M, Chan R G, De Silva T S, Mann E T, Russell J A, Spangler W H. Efficacy of exotic control strategies for restoring coastal prairie grasses. Invasive Plant Science and Management, 2014, 7(4) : 590-598.

[16] Rodriguez A M, Jacobo E J. Glyphosate effects on seed bank and vegetation composition of temperate grasslands. Applied Vegetation Science, $2013,16(1): 51-62$.

[17] Maggi F, La Cecilia D, Tang F H M, McBratney A. The global environmental hazard of glyphosate use. Science of the Total Environment, 2020, 717: 137167 .

[18] Weidlich E W A, Flórido F G, Sorrini T B, Brancalion P H S. Controlling invasive plant species in ecological restoration: a global review. Journal of Applied Ecology, 2020, 57(9): 1806-1817.

[19] Milakovic I, Fiedler K, Karrer G. Management of roadside populations of invasive Ambrosia artemisiifolia by mowing. Weed Research, 2014, 54 (3) : 256-264.

[20] Wilson M V, Clark D L. Controlling invasive Arrhenatherum elatius and promoting native prairie grasses through mowing. Applied Vegetation Science, 2001, 4(1): 129-138.

[21] Ramula S. Annual mowing has the potential to reduce the invasion of herbaceous Lupinus polyphyllus. Biological Invasions, 2020, 22( 10): 3163-3173.

[22] Espeland E K, Muscha J M, Scianna J, Kilian R, West N M, Petersen M K. Secondary invasion and reinvasion after Russian-olive removal and revegetation. Invasive Plant Science and Management, 2017, 10(4) : 340-349.

[23] Tognetti P M, Chaneton E J. Community disassembly and invasion of remnant native grasslands under fluctuating resource supply. Journal of Applied Ecology, 2015, 52(1): 119-128.

[24] 谭芳林, 林贻卿, 肖华山, 潘辉, 崔丽娟, 黄丽, 林捷, 罗美娟, 乐通潮, 罗彩莲. 不同时期刈割对互花米草生长影响的研究. 湿地科学, $2010,8(4): 379-385$.

[25] 唐龙. 刈割、淹水及芦苇替代综合控制互花米草的生态学机理研究 $[D]$. 上海: 复旦大学, 2008.

[26] 袁琳, 张利权, 肖德荣, 张杰, 王睿照, 袁连奇, 古志钦, 陈䂀, 平原, 祝振昌. 刈割与水位调节集成技术控制互花米草 (Spartina alterniflora). 生态学报, 2008, 28(11): 5723-5730.

[ 27] Major W W, Grue C E, Grassley J M, Conquest L L. Mechanical and chemical control of smooth cordgrass in Waillapa Bay, Washington. Journal of Aquatic Plant Management, 2003, 41: 6- 12.

[28] Gao Y, Tang L, Wang J Q, Wang C H, Liang Z S, Li B, Chen J K, Zhao B. Clipping at early florescence is more efficient for controlling the invasive plant Spartina alterniflora. Ecological Research, 2009, 24(5): 1033-1041.

[29] Zhao C Y, Li J S, Zhao X J. Mowing plus shading as an effective method to control the invasive plant Spartina alterniflora. Flora, 2019, 257: 151408 .

[30］赵相健, 李俊生, 柳晓燕, 宫璐, 赵彩云. 刈割加遮荫对互花米草生长和存活的影响. 广西植物, 2017, 37 (3) : 303-307.

[31］赵相健, 柳晓燕, 宫璐, 邓贞贞, 白加德, 李俊生, 赵彩云. 刈割加遮荫综合治理互花米草( Spartina alterniflora). 生态学杂志, 2014, 33 (10) : 2714-2719.

[32] 谢宝华, 韩广轩. 外来人侵种互花米草防治研究进展. 应用生态学报, 2018, 29(10): 3464-3476.

[33] 张丽平, 周亚圣, 刘君成, 尤丽萍, 王韧, 蔡金标, 游巍斌, 何东进. 遮荫对刈割互花米草生物量及渗透调节物质的影响. 西南林业大学 学报, 2019, 39(4): 142-148.

[34］张畑森. 覆盖对互花米草生理特性和生长的影响. 福建林业科技, 2016, 43(3)：72-75.

[35] 赵彩云, 白加德, 柳晓燕, 李俊生. 互花米草人侵对广西北海光滩大型底栖动物群落的影响. 环境科学研究, 2015, 28(3): 377-383.

[36] 仇乐, 刘金娥, 陈建琴, 王国祥, 常青. 互花米草扩张对江苏海滨湿地大型底栖动物的影响. 海洋科学, 2010, 34(8): 50-55.

[37] 赵秋毅. 泉州湾红树植物秋茄治理互花米草的生理生态效应研究 [D]. 厦门: 集美大学, 2019.

[38] 谷兴华. 无瓣海桑对互花米草地土壤因子变化影响的初步研究 $[\mathrm{D}]$. 长沙: 中南林业科技大学, 2010.

[39］覃盈盈. 红树林生境中互花米草的生态学研究 [D ]. 桂林: 广西师范大学, 2009. 